\title{
Prediction of skin cancer occurrence by ultraviolet solar index
}

\author{
MIGUEL RIVAS $^{1}$, ELISA ROJAS $^{1}$ and GLORIA M. CALAF ${ }^{2,3}$ \\ ${ }^{1}$ Department of Physics, Faculty of Science; ${ }^{2}$ Institute for Advanced Research, University of Tarapacá, Arica, Chile; \\ ${ }^{3}$ Center for Radiological Research, Columbia University Medical Center, New York, NY, USA
}

Received November 8, 2011; Accepted January 13, 2012

DOI: $10.3892 / \mathrm{ol} .2012 .580$

\begin{abstract}
An increase in the amount of solar ultraviolet light that reaches the Earth is considered to be responsible for the worldwide increase in skin cancer. It has been reported that exposure to excessive levels of solar ultraviolet light has multiple effects, which can be harmful to humans. Experimental ultraviolet light measurements were obtained in several locations in Chile between 2006 and 2009 using wide-band solar light Biometer YES, calibrated according to World Meteorological Organization (WMO) criteria and integrated into the National Meteorological Center of Chile ultraviolet network (DMC). The aim of this study was to determine skin cancer rates in relation to experimental data accumulated during one year of studying the solar ultraviolet index in Chile, in order to explain the possible effect of radiation on skin cancer. The rate of skin cancer per 100,000 persons was considered in Arica, Santiago, Concepción and Valdivia and extrapolated to other cities. Results of the present study showed that the incidence of skin cancer was markedly correlated with accumulative ultraviolet radiation, and rates of skin cancer could be extrapolated to other locations in Chile. There is a steady increase in the rate of skin cancer in cities located nearest to the equator (low latitude) that receive greater accumulated solar ultraviolet radiation, due to the accumulative effects of this type of radiation on the skin. It can be concluded that Arica is a city at sea level that receives higher levels of ultraviolet solar radiation than other locations, which may explain the higher prevalence of skin cancer in the population of this location, compared with other cities in Chile.
\end{abstract}

\section{Introduction}

An increase in the amount of ultraviolet solar light that reaches the Earth is considered to be responsible for the worldwide increase in skin cancer. This excessive solar ultraviolet (UV) radiation exposure led to the premature mortality of approximately 60,000 individuals in the year 2000 (1). Human exposure

Correspondence to: Dr Gloria M. Calaf, Instituto de Alta Investigación, Universidad de Tarapacá, Calle Antofagasta \#1520, Arica 8097877, Chile

E-mail: gmc24@columbia.edu

Key words: ultraviolet light index, skin cancer to excessive UV radiation causes a number of detrimental health effects, such as sunburn, sun-related eye disorders, photo aging, immune suppression and skin cancer. Skin cancer has been associated with prolonged exposure to UV. It has been reported that excessive levels of solar ultraviolet A (UVA) (320-400 nm) and solar ultraviolet B (UVB) have multiple effects, which can be harmful to humans (2-5). The quantity of light that reaches the earth is strongly dependent on the ozone layer (6), with absorption increasing with shorter wavelengths. UVA light that has high atmospheric transmission, while short-wavelength solar ultraviolet C (UVC) (100-290 nm) is completely absorbed and does not reach the surface of the earth. A small amount of solar UVB (290-320 nm) light reaches the surface of the earth and can be more dangerous to health than other UV light types. The amount of UVB that reaches the earth surface varies with altitude, latitude, albedo and ozone layer (7). People who receive more UVB exposure at noon have an increased skin cancer risk. Since skin cancer in fair-skinned individuals has shown a marked annual increase worldwide, it is crucial to determine the UV doses that people are exposed to around the world throughout their lives. In countries with fair-skinned populations, such as the United States, skin cancer is the most commonly diagnosed of all types of cancer. It has been reported that there is likely to be over one million new cases of skin cancer in 2002 (8).

UV doses reportedly increased with high altitude and decreased latitude. Moreover, the majority of indoor-working adult Europeans are exposed to $10,000-20,000 \mathrm{~J} / \mathrm{m}^{2}$ per year. Americans are exposed to $20,000-30,000 \mathrm{~J} / \mathrm{m}^{2}$ per year and Australians are estimated to be exposed to $20,000-50,000 \mathrm{~J} / \mathrm{m}^{2}$ per year (excluding vacations, which may increase the dose by $30 \%$ or more). The outdoor UV doses are weighted for different biological effects, the time that people spend outdoors, their ambient exposures, and the terrestrial and personal UV doses of adult outdoor and indoor workers, as well as children and adolescents around the world. The association between high UV levels with decreased latitude has been shown to increase melanoma cancer incidence in different racial and ethnic populations in a national data set (8). The data indicated that the melanoma incidence was associated with increased UV and lower latitude only in non-Hispanic whites. Latitude also had a significant correlation with incidence only in non-Hispanic whites. A substantial portion of the variance in registry incidence in non-Hispanic whites could be explained by the UV levels in this location, and it was concluded that melanoma incidence was associated with increased UV and lower latitude 
Table I. Rate of skin cancer in relation to latitude and linear fit of rate of incidence of skin cancer and accumulated ultraviolet solar index per year obtained in several cities in Chile per 100,000 persons.

A, Rate of skin cancer per 100,000 persons in relation to latitude.

\begin{tabular}{|c|c|c|c|c|}
\hline City & Latitude & $\begin{array}{l}\text { Rate of skin cancer } \\
\text { per } 100,000 \text { persons }\end{array}$ & Author & Year \\
\hline Arica & $18^{\circ} 18^{\prime}$ & 31.4 & Rivas et al & 2009 \\
\hline Santiago & $33^{\circ} 26^{\prime}$ & 21.7 & Zemelman et al & 2001 \\
\hline Concepción & $36^{\circ} 46^{\prime}$ & 19.3 & Muñoz & 2003 \\
\hline Valdivia & $39^{\circ} 48^{\prime}$ & 13.8 & Moreno et al & 2007 \\
\hline
\end{tabular}

B, Linear fit of a) Rate of incidence of skin cancer per 100,000 persons and b) accumulated ultraviolet solar index per year obtained in several cities of Chile.

\begin{tabular}{|c|c|c|c|c|}
\hline City & Latitude (grade) & UVB (DMC) & $\begin{array}{c}\text { Skin cancer incidence: } \\
\text { Linear fit }\end{array}$ & $\begin{array}{l}\text { Accumulated ultraviolet solar } \\
\text { index per year: Linear fit }\end{array}$ \\
\hline Arica & 18.3 & 117.5 & $32.0 \pm 0.7$ & $107 \pm 10$ \\
\hline Iquique & 20.5 & 90.0 & 30.3 & $103 \pm 15$ \\
\hline Antofagasta & 23.5 & 88.3 & 28.1 & $97 \pm 9$ \\
\hline Santiago & 33.4 & 70.0 & $20.7 \pm 0.8$ & $77 \pm 7$ \\
\hline Valparaíso & 32.9 & 76.3 & 21.0 & $78 \pm 1$ \\
\hline Concepción & 36.8 & 68.3 & $18.1 \pm 1.1$ & $70 \pm 2$ \\
\hline Puerto Montt & 41.4 & 61.7 & 14.7 & $60 \pm 1$ \\
\hline Cohayque & 45.6 & 61.7 & 11.5 & $52 \pm 10$ \\
\hline Punta Arenas & 53.0 & 60.0 & 6.0 & $37 \pm 5$ \\
\hline
\end{tabular}

UVB, ultraviolet B; DMC, National Meteorological Center of Chile ultraviolet network.

only in non-Hispanic whites. However, there was no evidence to support the association between UV exposure and melanoma incidence in black or Hispanic populations (9).

Measurements of occupational and total sun exposure appear to predict melanoma predominantly at low latitudes. The risk variation by tumor site and latitude was investigated in previous studies, in which it was found that melanoma risk was associated with sun exposure $(10,11)$. In their study, Chang et al (11) performed a pooled analysis of 15 case-control studies (5,700 melanoma cases and 7,216 controls), correlating patterns of sun exposure, sunburn and solar keratoses with melanoma risk. The results indicated that recreational sun exposure was a risk factor for melanoma on the trunk and limbs, but not on the head and neck across latitudes. Occupational sun exposure was associated with risk of melanoma on the head and neck at low latitudes. Total sun exposure was associated with increased risk of melanoma on the limbs at low latitudes, but not at other body sites or other latitudes. Sunburn in childhood for melanoma on the trunk, limbs, and head and neck showed little variation across latitudes. Chang et al also reported that the presence of head and neck solar keratoses was associated with an increased risk of melanoma on the head and limbs (11).

UV solar radiation exposure, latitude and skin cancer have led to increasing interest in the prevention of sun overexposure as more knowledge of photo-protection methods and UV levels becomes available. The solar ultraviolet index (UVI) is a well- known tool recommended by the World Health Organization (WHO) to avoid the harmful effects of UV sunlight. UVI forecasts are provided by numerous national meteorological services, but local UVI measurements are able to provide a more realistic and appropriate evaluation of UV levels. In this study, the experimental value of maximal daily values of UVI accumulated during one year was calculated using UVI experimental data of the UVB network of DMC under a cooperation agreement. The aim of the present study was to correlate the accumulated solar UVI and skin cancer prediction per 100,000 inhabitants in various locations in Chile.

\section{Materials and methods}

A national UVB network exists in Chile that extends daily public bulletins (12) to the population regarding the daily value of UVI measurements for the country. The measurements were classified according to the WHO parameters and were obtained and calibrated in accordance with the regulations of the World Meteorological Organization. In the city of Arica, experimental measurements were obtained using a wide band solar light Biometer YES, according to an agreement between Tarapacá University and the Meteorological Center of Chile from 2006 to 2009. To explain the possible effect of UV solar radiation on skin cancer, pathological reports were considered in four locations reported in the literature, including 


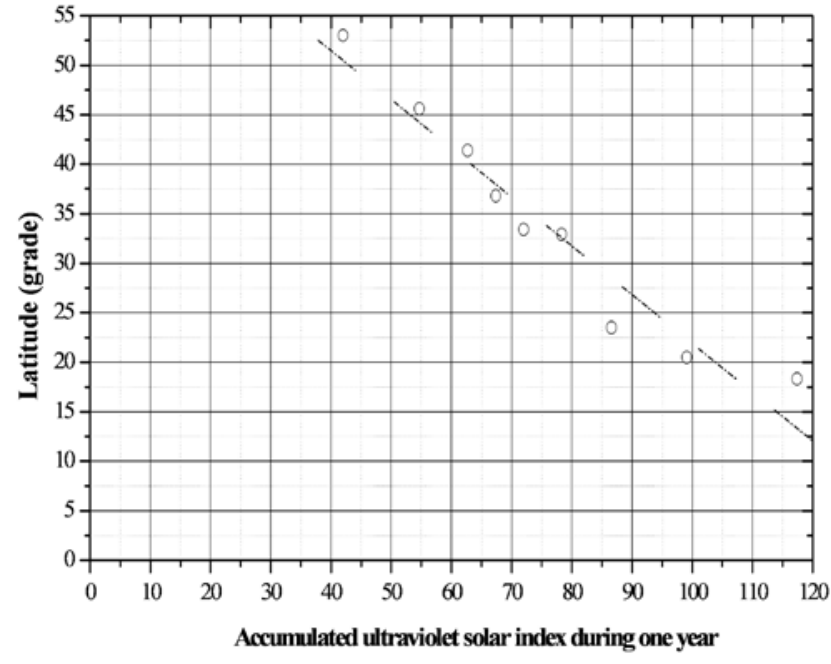

Figure 1. Experimental data of accumulated ultraviolet solar index during one year versus latitude (grades) in nine locations of Chile. The resulting linear fit equation is: $\mathrm{LAT}=71.7-0.493 \cdot(\mathrm{UVI})$.

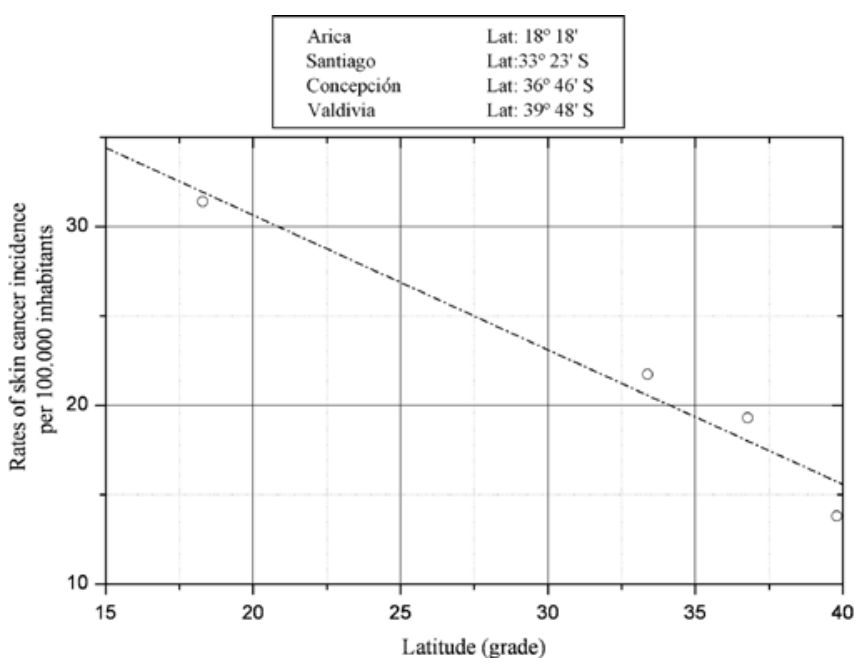

Figure 2. Rates of incidence of skin cancer per 100,000 persons (R) registered in four locations of Chile versus latitude (LAT). Data were collected during the 1993-2007 period. The resulting linear fit equation is: $\mathrm{R}=45.718-0.753 \cdot(\mathrm{LAT})$.

Arica [2000-2007, Rivas et al (13,14)], Santiago [1992-1998, Zemelman et al (15)], Concepción [2007, Muñoz (16)] and Valdivia [1997-2007, Moreno and Lopehandia (17)].

The study was conducted with patient approval according to grant regulations.

\section{Results}

The present study investigated the level of solar UVI accumulated in relation to skin cancer in various locations in Chile. In this study, experimental values of accumulated solar UVI for one year were correlated with skin cancer rates per 100,000 persons in four locations in order to obtain the behavior of these variables and extrapolate to other locations of the country in which UV measurements had been obtained.

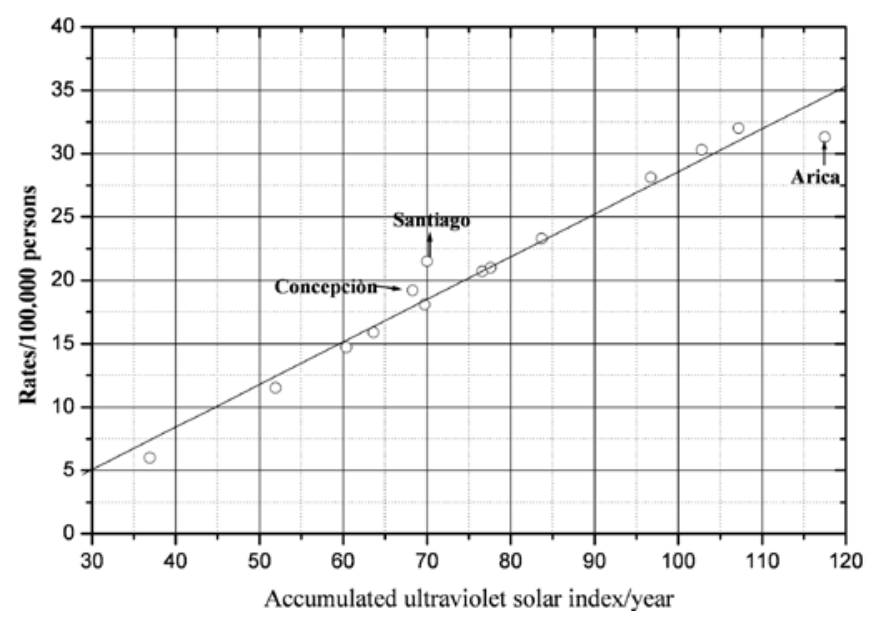

Figure 3. Rates of incidence of skin cancer per 100,000 persons (R) versus accumulated ultraviolet solar index (UVI) when the results, obtained by the linear fit equations in Figs. 1 and 2, were applied to nine locations of Chile. The resulting linear fit equation is: $\mathrm{R}=5.019+0.336 \cdot(\mathrm{UVI})$.

Table IA shows the rate of skin cancer per 100,000 in relation to latitude. Table IB indicates the skin cancer incidence of three cities (Arica, Santiago and Concepción), which provided information for determining the linear fit rate of incidence of skin cancer per 100,000 people and accumulated solar UVI per year in several cities in Chile. The results indicated that the accumulated solar UVI was 2.82 times greater in Arica than Punta Arenas, fluctuating from 117.5 to 41.7. Fig. 1 shows the corresponding linear fit of the latitude (grades) versus accumulated solar UVI for the period 2006-2009. The resulting linear fit equation is: LAT $=71.17-0.493 \cdot(\mathrm{UVI})$, where LAT is latitude of the city location in degrees and (UVI) is the accumulated UV solar index during one year. Fig. 2 shows the rates of incidence of skin cancer versus latitude registered in four locations of Chile. The resulting linear fit equation is: $\mathrm{R}=45.72-0.75 \cdot(\mathrm{LAT})$, where $(\mathrm{R})$ is the rate of incidence of skin cancer and (LAT) is the latitude of location. Fig. 3 shows the rates of skin cancer incidence versus the accumulated solar UVI and these data were obtained when we applied the results obtained by the linear fit equations obtained in Figs. 1 and 2. Our results compared experimental points in Fig. 3 that corresponded to Arica, Santiago and Concepción.

\section{Discussion}

Recreational sun exposure and sunburn are strong predictors of melanoma at all latitudes, whereas measurements of occupational and total sun exposure appear to predict melanoma predominantly at low latitudes. Grant (10) has investigated risk variation by tumor site and latitude. The results of that study showed that melanoma risk was correlated with sun exposure. Occupational sun exposure was associated with risk of melanoma on the head and neck at low latitudes. Total sun exposure was associated with increased risk of melanoma on the limbs at low latitudes, but not at other body sites or other latitudes. When sunburn in childhood associated with melanoma was considered on the trunk, limbs, and head and neck, little variation was found across latitudes. Since the 
presence of head and neck solar keratosis was associated with increased risk of melanoma on the head and limbs, the author concluded that melanoma risk at various body sites was associated with different amounts and patterns of sun exposure. Chang et al (11) also reported that melanoma risk at various body sites is associated with different amounts and patterns of sun exposure. Thus, sun exposure when outdoors and the resulting sunburn are strong predictors of melanoma at all latitudes. However, occupational and total sun exposure appear to predict melanoma largely at low latitudes.

Krishnamurthy (18) studied the correlation of melanomas from skin cancer registries in various parts of India with latitude, altitude, ozone levels and ultraviolet light exposure. The results revealed slightly negative associations of melanoma with latitude and statistically non-significant associations with atmospheric ozone levels. There was a positive association with UV solar light with melanoma incidence from four locations, and this was correlated in a parabolic curve with latitude. Geographic patterns of melanoma in Indians are similar to those in white Caucasians; therefore, ultraviolet light exposure may also be involved in the etiology of skin cancer in non-white Caucasians such as Indians.

Lee and Sotto (19) found that linked temporal and latitude changes were elevated with regards to incidence and mortality from melanoma of the skin. Additionally, these authors found that this process is slowing down in younger age groups in the United States. In many Causasian populations, including that of the United States, melanoma incidence and mortality rates increased according to proximity of residence to the equator. Variations with age in this gradient do not appear to have been examined. The effect of latitude on melanoma rates was examined and the results showed that this effect varied with age (19). Moreover, authors of that study found a decline from old age to youth in the effect of latitude for both incidence and mortality from melanoma of the skin in males, and for mortality in females. Furthermore, these changes in the relationship of latitude with age correlated with the changes in time trends with age.

Eklund and Malec (20) showed a linear correlation analysis between latitude and melanoma incidence rate in various regions, and revealed a decreasing incidence with increasing latitude. This result supports the hypothesis that UV irradiation is the predominant cause of melanoma. However, considerable deviations from the regression line were observed in certain regions. Moreover, a comparison between town and country indicated melanoma incidence increased with population density. This increase is not explained by over-diagnosis and thus is not in agreement with the working hypothesis of solar UV irradiation as the predominant cause of melanoma.

\section{Acknowledgements}

This study was supported by the grant UTA-Mayor 4721 (2011-2012) (MR and ER) and by Convenio de Desempeño, University of Tarapacá-Mineduc, Chile (GMC).

\section{References}

1. Lucas R, McMichael T, Smith W and Armstrong B: Solar ultraviolet radiation: global burden of disease from solar ultraviolet radiation. World Health Organization, Switzerland, pp250, 2006.

2. Jagger J: Solar UV Actions On Living Cells. Praeger, New York, 1985.

3. Elwood JM and Jopson J: Melanoma and sun exposure: an overview of published studies. Int J Cancer 73: 198-203, 1997.

4. Baron EA, Fourtanier A, Compan D, Medaisko C, Cooper KD and Stevens SR: High ultraviolet a protection affords greater immune protection confirming that ultraviolet A contributes to photo immune suppression in humans. J Invest Derm 121: 869-875, 2003

5. Van der Leun JC, De Gruijl FR, Tevini M and Worrest RC: Ultraviolet carcinogenesis: environmental effects of ozone depletion. Recent Results Cancer Res 128: 263-267, 1993.

6. Sánchez F: Consideraciones sobre la capa de ozono y su relación con el cáncer de piel. Revista Médica de Chile 134: 1185-1190, 2006 (In Spanish).

7. Madronich S, Mckenzie RI, Bjorn LO and Caldwell MM: Changes in biologically active ultraviolet radiation reaching the Earth's surface. J Photochem Photobiol 46: 5-19, 1998.

8. Godar DE: UV doses worldwide. Photochem Photobiol 81: 736-749, 2005.

9. Eide MJ and Weinstock MA: Association of UV index, latitude and melanoma incidence in nonwhite populations-US Surveillance, epidemiology and end results (SEER) Program 1992 to 2001. Arch Dermatol 141: 477-481, 2005.

10. Grant WB: Risk of internal cancer after diagnosis of skin cancer depends on latitude smoking status and type of skin cancer. Int J Cancer 124: 1741-1742, 2009.

11. Chang YM, Barrett JH, Bishop DT, et al: Sun exposure and melanoma risk at different latitudes: a pooled analysis of 5700 cases and 7216 controls. Int J Epidemiol 38: 814-30, 2009.

12. González M: Bulletin of ultraviolet solar radiation. Dirección Meteorológica de Chile, Climatología y Meteorología Aplicada 18: 18-24, 2009.

13. Rivas M, Araya MC, Durán V, Rojas E, Cortes J and Calaf GM: Ultraviolet light exposure and skin cancer in the city of Arica, Chile. Mol Med Rep 2: 567-572, 2009.

14. Rivas M, Araya MC, Caba F, Rojas E and Calaf GM: Ultraviolet light exposure influences skin cancer in association with latitude. Oncol Rep 25: 1153-1159, 2011.

15. Zemelman V, Roa V, Díaz C, Araya I, Zamalloa G and Faundez E: Aumento de la incidencia del Cáncer cutáneo en la Región Metropolitana. Revista Chilena de Dermatología 17: 180-185, 2001 (In Spanish).

16. Muñoz P: Incidencia de cáncer cutáneo, Hospital Regional de Concepción, VII Región (unpublished thesis). Thesis Degree in Dermatology, Concepción University, 2003.

17. Moreno M and Lopehandia J: Cáncer de piel en Valdivia entre los años 1995 y 2000. Revista Chilena de Dermatología 23: 184-186, 2007 (In Spanish).

18. Krishnamurthy S: The geography of non-ocular malignant melanoma in India: its association with latitude, ozone levels and UV light exposure. Int J Cancer 51: 169-172, 1992.

19. Lee JA and Scotto J: Melanoma: linked temporal and latitude changes in the United States. Cancer Causes Control 4: 413-418, 1993.

20. Eklund G and Malec E: Sunlight and incidence of cutaneous malignant melanoma. Effects of latitude and domicile in Sweden. Scand J Plast Reconstr Surg 12: 231-241, 1978. 